\title{
Intermédialités
}

Histoire et théorie des arts, des lettres et des techniques

Intermediality

History and Theory of the Arts, Literature and Technologies

\section{Muliats et Avant les rues : la politique de l'oeuvre hétérolinguale}

\section{Karina Chagnon}

Numéro 27, printemps 2016

traduire

translating

URI : https://id.erudit.org/iderudit/1039811ar

DOI : https://doi.org/10.7202/1039811ar

Aller au sommaire du numéro

Éditeur(s)

Revue intermédialités (Presses de l’Université de Montréal)

ISSN

1920-3136 (numérique)

Découvrir la revue

Citer cet article

Chagnon, K. (2016). Muliats et Avant les rues : la politique de l'oeuvre

hétérolinguale. Intermédialités / Intermediality, (27).

https://doi.org/10.7202/1039811ar
Résumé de l'article

Ce texte propose d'étudier deux oeuvres récentes, la pièce de théâtre Muliats des Productions Menuentakuan (2016) et le film Avant les rues de Chloé Leriche (2016). Ces oeuvres collectives de traduction et de non-traduction permettent une réflexion sur l'appropriation de différents dispositifs intermédiaux en vue de faire exister, dans l'espace public, les langues innue et atikamekw dans une intimité hétérolinguale avec le français québécois. 


\title{
Muliats et Avant les rues : la politique de l'œuvre hétérolinguale
}

\author{
KaRINA ChAGNON
}

epuis quelques années maintenant, et plus particulièrement depuis la parution du rapport de la Commission de vérité et réconciliation (CVR) en 20I5, la société canadienne se voit interpellée sur les politiques dévastatrices des pensionnats ${ }^{\mathrm{I}}$ et par le mouvement de résurgence politique et culturelle autochtone. L'articulation du concept de « génocide culturel » dans le rapport de la CVR, qui définit le traitement réservé aux enfants autochtones dans les pensionnats pendant plus de I5O ans, a eu pour effet de produire une conscience accrue des conséquences actuelles de cette politique gouvernementale d'assimilation. En retour, cette conscience grandissante nourrit le mouvement de résurgence politique qui s'élève notamment contre le linguicide ${ }^{2}$ et la minorisation des langues autochtones. La résistance à la violence coloniale d'élimination des langues autochtones et des modes de vie qui leur correspondent se manifeste de plus en plus dans les arts au Québec.

Ce texte propose d'étudier deux œuvres récentes, la pièce de théâtre Muliats $^{3}$ (2016) des Productions Menuentakuan et le film Avant les rues (2016) de Chloé

I Voir « Conclusions de la CVR », Commission de vérité et réconciliation du Canada, 2015.

${ }^{2}$ Lorena Fontaine de l'Université du Manitoba qualifie l'attaque contre les langues autochtones de «linguicide». Lorena Fontaine, «Ideas from the Trenches-Undoing Linguicide », entrevue radiophonique réalisée avec Paul Kennedy dans le cadre de l'émission Ideas sur les ondes de CBC/Radio-Canada, diffusée le iq juillet 2016, http://www.cbc.ca/radio/ideas/ideas-from-the-trenches-undoing-linguicide-I.3525609 (consultation le I4 octobre 20I6).

3 Muliats, scénario composé par Charles Bender, Marco Collin, Xavier Huard, Natasha Kanapé et Christophe Payeur, mise en scène de Xavier Huard, Productions Menuentakuan, Théâtre Denise-Pelletier, Montréal, du 2 au 20 février 2016. Je tiens à remercier Xavier Huard, metteur en scène des Productions Menuentakuan, d'avoir généreusement partagé avec moi le texte inédit de la pièce de théâtre Muliats. 
Leriche. Ces œuvres permettent une réflexion sur l'appropriation de différents dispositifs intermédiaux en vue de faire exister, dans l'espace public, les langues innue et atikamekw dans une intimité hétérolinguale avec le français québécois (pour une carte des langues autochtones $\left.{ }^{4}\right)$.

L'existence de ces langues autochtones dans l'espace culturel francophone engage un dialogue que je qualifierais de politique avec le monolinguisme des spectateurs québécois francophones. En plus de rendre compte des conséquences de l'imposition du français colonial, les œuvres se démarquent par leur hétérolinguisme, c'est-à-dire leur tissage, dans la distance, de langues minorisées avec la langue dominante. On peut dès lors se demander : en quoi ces œuvres, caractérisées à la fois par la traduction et la non-traduction, nous invitent-elles à repenser nos rapports politiques entre colons et colonisés, et à contester des formes de représentation stéréotypées et réductrices ? Comment ces échanges entre langue dominante et langues minorisées nous permettent-ils de repenser notre appartenance à la communauté ? Enfin, quelles ont été les démarches d'appropriation des médias que sont le théâtre et le cinéma pour faire exister les langues innue et atikamekw ?

Mon hypothèse est que les œuvres proposent des «matérialités de la communication » qui, comme l'explique Éric Méchoulan, « font partie du travail de signification et de références $»$. Ces objets ont pour fonction la «fabrication de présence » et de «modes de résistance » dans un contre-discours élaboré collectivement par les artistes autochtones eux-mêmes ${ }^{6}$. En ce sens, l'intermédialité propre au théâtre et au cinéma autochtones nous amène à remettre en question les relations de pouvoir à travers ce que Christian Biet et Christophe Triau appellent la « séance théâtrale », une relation caractérisée par une dialectique active, jamais résolutive, mais qui opère plutôt dans la tension7. Dans Muliats et Avant les rues, le je est un Autochtone: un être complexe, contemporain, et qui n'a nul besoin de l'humanisme colonial pour « conserver » son savoir ou sa culture. Bruno Cornellier

4 Pour une carte des communautés autochtones de la région, voir « Cartes des communautés autochtones du Québec », Secrétariat aux affaires autochtones, 2 février 2016, http://www.versuntraite.gouv.qc.ca/nations/cartes_communautes.htm (consultation le I8 février 20I7).

5 Éric Méchoulan, «Intermédialités: Le temps des illusions perdues », Intermédialités, $\mathrm{n}^{\circ} \mathrm{I}$ « naitre », printemps 2003, p. IO.

${ }^{6}$ Ibid., p. 22.

7 Christian Biet et Christophe Triau, «La comparution théâtrale. Pour une définition esthétique et politique de la séance $\gg$, Tangence, $\mathrm{n}^{\circ} 88,2008$, p. 3I-32. 
explique que les colonies de peuplement du Québec et du Canada s'arrogent le pouvoir de désignation de ce que constitue l'« Indien ». Ainsi, parmi les (mé)représentations cinématographiques et télévisuelles se retrouve le projet historique de commensuration des différences d'une « race » ou d'un territoire «nouveau ». La représentation indienne qui surgit est celle d'une «vie nue qui demande à être sauvée, protégée, déterrée, conservée sous un présentoir muséal, sous la tutelle et le protectorat du souverain, avant d'être invitée, en fin de parcours, à s'intégrer en tant que "notre" égale au sein du corps politique ${ }^{8} \gg$. En revanche, Muliats et Avant les rues abordent de front les questions de la consommation des cultures exotiques, la relocalisation discursive en milieu urbain et l'imposition coloniale de la catégorisation raciale.

Ce je s'exprime autant en langues innue ou atikamekw que française, d'où l'intérêt d'explorer les œuvres pour leur portée hétérolinguale. De fait, les figures présentées dans les œuvres déconstruisent les dichotomies entre le commun et l'étranger à travers le concept d'intimité hétérolinguale élaboré par Jon Solomon, qui définit cette dernière comme «une forme éthique où l'on reconnaît dans l'adresse l'élément de distance inhérent à toute relation sociale $\gg 9$. Le caractère collectif des deux œuvres à l'étude, qui s'apparente, au sens deleuzien, à un agencement collectif d'énonciation, permet de faire le pont avec les récentes recherches en méthodologie de décolonisation $^{\mathrm{IO}}$. Cette méthodologie, qui s'articule principalement en langue anglaise, se veut une réponse à la longue histoire de colonisation par différents domaines scientifiques, dont l'anthropologie et l'ethnographie. Dans les mécanismes de création des œuvres, une place centrale semble être accordée à la réciprocité, une composante centrale de la méthodologie de décolonisation. Dans le cadre de Muliats et d'Avant les rues, l'emphase mise sur le travail en collectivité, mêlant Autochtones et nonAutochtones, suggère que le travail de création a pu s'inspirer d'un intérêt pour la réflexion, les échanges, les débats et les discussions entre les participants.

${ }^{8}$ Bruno Cornellier, La «chose indienne». Cinéma et politiques de la représentation autochtone dans la colonie de peuplement libérale, Québec, Éditions Nota bene, 2015, p. I8-19. En revanche, Muliats et Avant les rues abordent de front les questions de la consommation des cultures exotiques, la relocalisation discursive en milieu urbain et l'imposition coloniale de la catégorisation raciale.

9 Jon Solomon, « Traduction, violence et intimité hétérolinguale », Rada Iveković (dir.), Translating Violence, Transversal, vol. II, $\mathrm{n}^{\circ} 7$, 2007, http://eipcp.net/transversal/Iro7/ solomon/fr (consultation le 3 mai 2016).

Io Voir Eve Tuck et Wayne K. Yang, « Decolonization is not a Metaphor », Decolonization, Indigeneity, Education ES Society, vol. I, $\mathrm{n}^{\circ} \mathrm{I}, 2 \mathrm{I} 2$, p. I-40. 


\section{LA NON-TRADUCTION COLLECTIVE ET LE CONTRE-POUVOIR}

La traduction collective est au cour de la pièce Muliats et du film Avant les rues, mais de façon tout à fait opposée : la pièce de théâtre pourrait être qualifiée de non-traduction, où se mélangent des dialogues en français et en innu, tandis que le film propose un effet de double traduction. Majoritairement interprété en langue atikamekw, le film Avant les rues est sous-titré en français tout en comprenant des dialogues qui mélangent l'atikamekw et le français québécois. Il faut ici rappeler que la littérature théâtrale québécoise s'est instituée, depuis la fin des années 1960 comme modalité doxologique de l'identité nationale. Dans son étude sur la sociocritique de la traduction du théâtre québécois (I968-I988), la traductologue Annie Brisset explique que la traduction a été employée par les dramaturges - pensons à Michel Tremblay et Michel Garneau - pour élever le langage vernaculaire québécois afin que celui-ci « accède au statut de langage littéraire à la place du français de France ${ }^{\mathrm{II}}$ ». Plutôt que de traduire pour transmettre le discours de l'Étranger, la traduction identitaire au Québec a eu tendance à mettre l'accent sur la langue d'arrivée, utilisant le discours des textes étrangers pour «cautionner son propre discours, celui de l'émancipation nationale ${ }^{\mathrm{I} 2} \gg$. Si l'identité québécoise s'est imposée comme projet politique grâce, entre autres, à la traduction au théâtre et au cinéma, elle l'a fait au détriment du « mineur » en son sein, dont le « mineur autochtone », qui n'a, jusqu'à récemment, pas eu accès à ces modalités d'expression. La «déterritorialisation », au sens deleuzien, cette tentative de miner l'expression du contenu par l'emploi de langues inconnues par la société dominante, permet de fuir et de déstabiliser le monopole de la langue française, coloniale. On peut dès lors se comprendre sous son aspect médial : les formes théâtrale et cinématographique doivent être investies et conquises pour que la rencontre des langues ait lieu.

\section{MULIATS ET LA COMPARUTION THÉÂtRALE}

Dans le texte de présentation de Muliats, on peut lire: « La pièce sonde le gouffre, trop souvent ignoré, qui existe entre deux nations à la recherche de repères ${ }^{\mathrm{I} 3} \gg$. Dans sa dimension politique, l'œuvre met en scène l'arrivée de Shaniss à Muliats

\footnotetext{
II Annie Brisset, Sociocritique de la traduction. Théâtre et altérité au Québec (1968-1988), Montréal, Le Préambule, coll. « L’Univers des discours », I990, p. 36.

${ }^{12}$ Ibid., p. 3 I2.

${ }_{13}$ Muliats, livret de présentation, Productions Menuentakuan et Théâtre Denise-Pelletier, du 2 au 20 février 2016, Salle Fred-Barry, Montréal, p. 4.
} 
- Montréal en langue innue. La pièce, qui ne possède d'autre décor qu'une énorme table en bois au milieu de la scène, produits certes des effets de sens à partir des dialogues en français, mais également par des chants accompagnés de tambour et la récitation de poèmes en langue innue. Tous ces objets forment des nouds de relations qui interpellent de diverses façons les spectateurs autochtones et non autochtones. Le personnage principal, Shaniss, est métis, né d'une mère innue et d'un père québécois. Il se retrouve en conflit avec son frère qui, lui, possède deux parents innus. En arrivant à Montréal, Shaniss fait le choix de changer son nom pour celui de Charles afin de « passer » pour un Québécois plutôt qu'un Autochtone. Mais il apparaît rapidement, à la rencontre de son colocataire, qu'un gouffre politique et culturel précède leurs rapports :

Christophe : T'arrives d'où comme ça?

Charles : Mashteuiatsh.

Christophe : Oh, ok! Ok! C’est combien d'heures environ...?

Charles : 5 ou 6 heures...

Christophe : Ayoye, ok ! Donc pour toi, en ce moment, y'est genre... 3 h du matin !!! Tu dois être fatigué !!!

Charles : Hein?

Christophe : Ben, le décalage horaire... tu dois être fatigué...

Charles est surpris.

Charles : Non, non... 5-6 heures de bus. Mashteuiatsh, c'est au Saguenay, Pointe-Bleue... au Lac-Saint-Jean...

Christophe: Oh boy! Ahahahah! Eille, s'cuse-moi, je connais pas vraiment mon Québec... je veux dire, en dehors de Montréal. (Temps.) Pis tu faisais quoi là-bas ?

Charles : Je viens de là.

Christophe : Ah ouain ?

Temps.

Christophe : Donc tu es... Je veux dire... Es-tu... ?

Charles : Je suis innu.

Christophe : It ?

Christophe : Inuit ?

Charles : Non, pas inuit, innu, c'est pas la même chose, vraiment pas.

Christophe : Ouain... (Il rit sec.) S'cuse. J'voulais dire... mais t'es-tu ?... j’veux dire... 
Charles: Ouais... (Malaise presque imperceptible. Christophe ne le remarque même pas.) J'suis autochtone là, avec la carte pis tout ${ }^{\mathrm{I} 4}$.

La rencontre entre les deux personnages donne lieu à une multiplication de malaises créés par les maladresses de Christophe, notamment lorsque celui-ci offre une coiffe cérémoniale du peuple lakota en cadeau à Shaniss. La séance, que Biet et Triau appellent la «comparution théâtrale », met à nu le lien social colonial dans ses

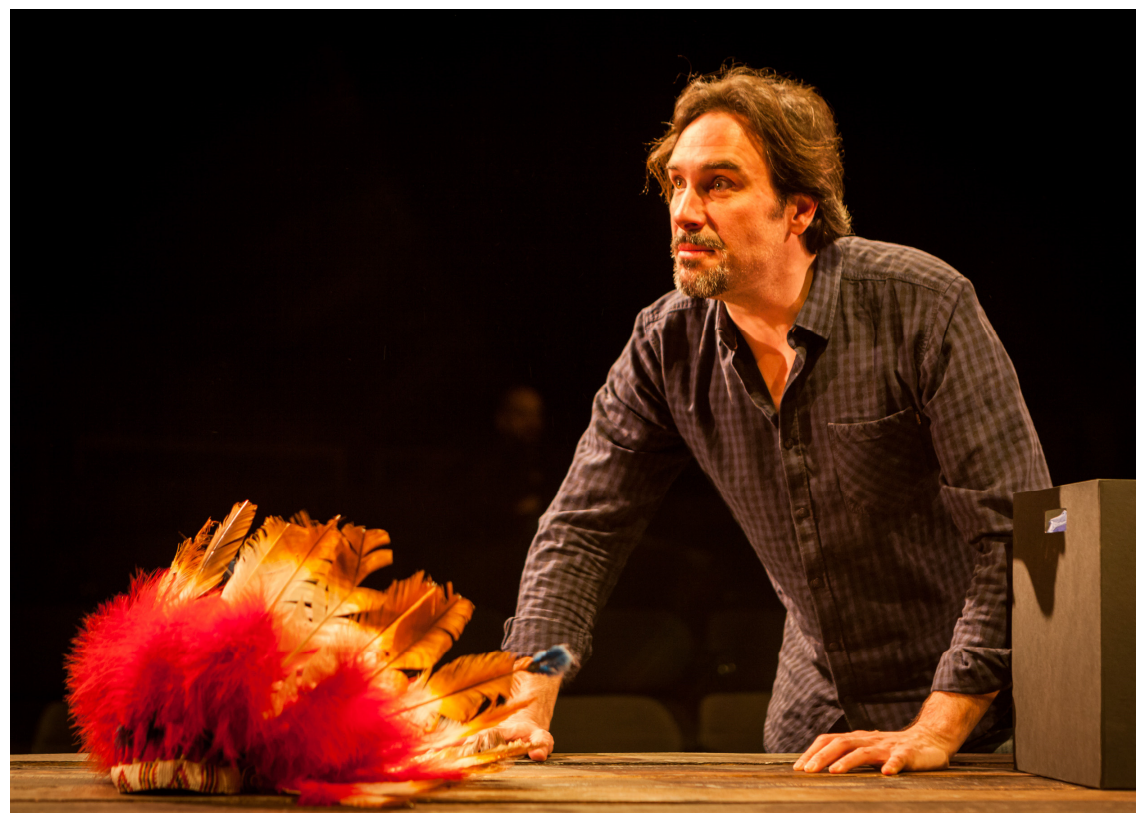

Fig. I. Shaniss et la coiffe. Muliats, Productions Menuentakuan et Théâtre Denise-Pelletier, 2016. Photo : Colin Earp-Lavergne.

différentes expressions (voir la figure I).

Les deux colocataires trouvent aussi des points de convergence, dont le poème de Michèle Lalonde «Speak White» (1968). Shaniss croit que l'auteure est autochtone alors qu'il lit un extrait du célèbre poème ${ }^{\mathrm{IS}}$.

À l'heure où le soleil s'en vient crever au-dessus des ruelles, mais pour vous dire oui que le soleil se couche oui

${ }^{14}$ Charles Bender et al., Muliats, texte inédit, Productions Menuentakuan, 2016, p. 4.

is Michèle Lalonde, «Speak White », Speak White, Montréal, L'Hexagone, 1974, cité dans Muliats, 2016, p. 6-7. 


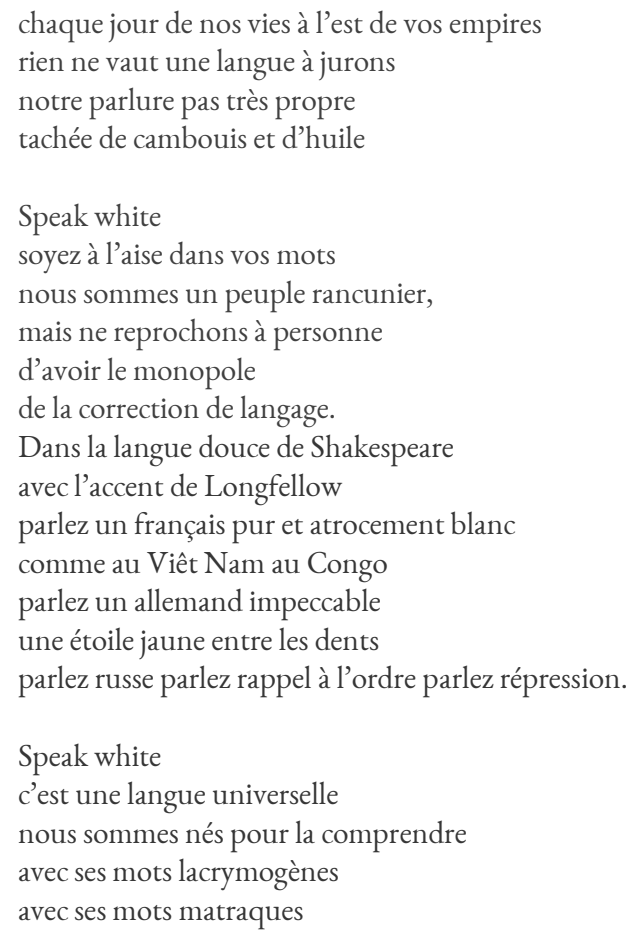

Charles : Hein ? (Temps.) Michelle Lalonde est pas autochtone? Christophe : Non.

Charles : Ben voyons, toi... Pourquoi elle a écrit ça d'abord si elle est pas autochtone ${ }^{16}$ ?

Shaniss, avec sa question, soulève l'enjeu de la domination du français par rapport aux langues autochtones au Québec. Le point de rencontre entre le français québécois et l'innu est cette «parlure pas très propre tachée de cambouis et d'huile », émergeant du statut mineur du français québécois qui se greffe au français colonial, normatif. En soulevant l'historicité de certains événements spectaculaires, dont la récitation orale de Michelle Lalonde, le texte théâtral évoque des temps et des lieux empruntés à l'histoire pour soulever des enjeux politiques actuels, c'est-à-dire la constitution d'une québécité dans la négation de l'autochtonie qui lui est à la fois antérieure et contemporaine. Biet et Triau écrivent à ce sujet :

[Le] phénomène qui s'élabore dans la séance [...], selon nous, pourrait être nommé « comparution ». On dira que, lors d'une séance théâtrale, comparaissent, les uns devant les autres et à travers des figurations hétérogènes et des postures différentes, les

\footnotetext{
${ }^{16}$ Charles Bender et al., Muliats, texte inédit, 2016, p. 6-7.
} 
acteurs, les spectateurs, les praticiens, mais aussi les personnages et les performeurs entre eux et devant les publics [...] Comparutions multiples qui figurent et induisent des jugements multiples, mettant à nu dans le milieu théâtral la nécessité du lien social en même temps que la nécessité, en en prenant conscience, de le perturber ou de le questionner. Il ne s'agit donc ici [...] d'une mise en pratique et en jeu des instances et des individus à l'intérieur d'un même lieu alors qu'ils ont conscience de leur(s) présence(s) et de leur co-présence afin qu'ils s'interrogent sur cette présence même. Comparution immédiate, parce que faite dans l'instant, dans l'éphémère de la séance, avec tous les risques que l'hétérogénéité des relations inscrites dans la séance suppose. Comparution médiate ou médiatisée, parce qu'à l'intérieur de la séance, il y a performance ou représentation d'un autre événement que le simple événement de rassemblement, parce qu'il y a $\ll$ art $»^{17}$.

À l'époque de la Révolution tranquille au Québec, le rôle de la langue vernaculaire était central dans la volonté d'affirmation politique. Mais presque un demi-siècle plus tard, bien que le projet souverainiste demeure inachevé, la langue française s'est institutionnalisée pour devenir la langue de la littérature, du théâtre et du cinéma. En somme, la langue est devenue celle du pouvoir, remplaçant la langue anglaise, tandis que les langues des peuples autochtones sont demeurées minoritaires. Ainsi, le choix de ne pas traduire les répliques en langue innue dans Muliats peut être compris comme un choix politique de non-communication, comme une volonté de faire vivre et de faire entendre la langue innue comme expression de résistance qui échappe à l'interprétation du public francophone et est réservée au public innu ${ }^{18}$.

Dans Muliats, le choix de ne pas traduire conduit à une nouvelle configuration du commun et de l'étranger. Le commun ici est innu : un seul personnage, Christophe, ne comprend pas la langue et devient, pour ainsi dire, l'étranger. À ce renversement des rapports de force, Christophe réplique par l'incompréhension et la victimisation. En réponse au trope usé de la contrainte entre l'assimilation nécessaire ou la disparition inévitable des Autochtones, Shaniss et son frère Marco invitent Christophe à s'intéresser à eux, dans leurs existences contemporaines et continues à leur territoire, en l'invitant à Mashteuiatsh. Tournée vers l'avenir, Muliats propose un espace commun où les différences, même inconciliables, peuvent être célébrées.

Alors que la bande-annonce, entièrement en langue innue, présente la langue comme une ambiance sonore qui s'associe à des paysages et à des visages muets, la

${ }^{17}$ Biet et Triau, 2008, p. 40-4I [je souligne].

${ }^{18}$ À ce titre, la bande-annonce, produite entièrement en langue innue, est révélatrice de la poétique et de la politique de l'œuvre. Voir « Muliats », Théâtre Denise-Pelletier, 2or6, http://www.denise-pelletier.qc.ca/spectacles/45/ (consultation le 20 décembre 2016). 
séance théâtrale nous plonge plus loin dans les enjeux politiques de la prise de parole. Paul Zumthor résume ainsi les enjeux de la performance orale :

Radicalement sociale, autant qu'individuelle, la voix, en transmettant un message, signale en quelque façon la manière dont son émetteur se situe dans le monde et à l'égard de l'autre à qui il s'adresse. La présence, dans un même espace, des participants de cet acte de communication, les met en position de dialogue (réel ou virtuel), engageant ici et maintenant, dans une action commune, leur totalité individuelle et sociale. L'écriture est inapte à produire de tels effets, sinon de façon indirecte et métaphorique ${ }^{19}$.

Les répliques en langue innue produisent deux effets : d'abord, elles interpellent un public qui n'a pas besoin de la langue française, qui s'en est libéré et est donc autonome; ensuite, elles démontrent les limites du monolinguisme du public francophone, son incapacité à saisir les réalités qui se situent en dehors de la norme coloniale. En somme, la pièce vient confondre les horizons d'attente des auditeurs. Pour reprendre les termes de Zumthor, les « points d'ancrage » des auditeurs, dans leur affectivité profonde et dans leurs fantasmes, dans leur idéologie, dans leurs habitudes personnelles, sont complètement bouleversés ${ }^{20}$. C'est une parole de résistance dans le non-acte qui surgit devant le public colonial. En ce sens, la noncommunication peut être un choix volontaire pour échapper à la violence de la domination par la traduction. Dans le cas de Muliats, on pourrait même voir la nontraduction comme un acte politique. La présence de l'hétérolinguisme dans la pièce parvient à opposer le caractère opprimé au caractère oppresseur de la langue.

\section{MULIATS ET L’AgENCEMENT COLLECTIF D’ÉNONCIATION}

Muliats est la première pièce de la compagnie Menuentakuan, mot innu qui pourrait se traduire par « prendre le thé en bonne compagnie, se dire les vraies choses et avoir du bon temps ${ }^{2 \mathrm{I}} \gg$. La pièce a été composée par un collectif composé d'une majorité d'Autochtones et de quelques allochtones, dont les comédiens de la pièce. Comme dans un agencement collectif d'énonciation au sens deleuzien, tout prend une valeur collective, que l'agencement émane d'un collectif de création ou encore d'une

I9 Paul Zumthor, « Oralité », Intermédialités, n I2, 2008, p. 182.

${ }^{20}$ « La fonction d'une poésie orale se manifeste par rapport à l'horizon d'attente des auditeurs. Indépendamment de tout jugement rationnel, et dans l'immédiateté de la communication, le texte entendu répond à une question que se pose l'auditeur : point d'ancrage du texte dans son affectivité profonde et ses fantasmes, dans son idéologie, dans ses habitudes personnelles ». Ibid., p. I9I.

${ }^{21}$ Muliats, livret de présentation, 2016, p. 3. 
parole individuelle : tout est énonciation collective, le champ politique a contaminé tout énoncé. C'est à la littérature que revient le rôle de l'énonciation collective, voire révolutionnaire : « [...] parce que la conscience collective ou nationale est souvent inactive dans la vie extérieure et toujours en voie de désagrégation ${ }^{22} \gg$. La machine littéraire prend le relais de la machine révolutionnaire parce qu'elle est la seule à être apte à remplir les conditions d'une énonciation collective. Pour transposer le discours en littérature théâtrale, il faudra y ajouter le rôle de la parole sur scène. Chiel Kattenbelt décrit, dans « Theatre as the Art of the Performer and the Stage of Intermediality », que, dans sa modalité dramatique, le théâtre se définit par l'actualité et la causalité de l'acte, comme si l'intensité de l'expérience et la réflexivité de la pensée étaient d'une plus grande importance que ses dimensions lyriques ou épiques ${ }^{23}$. Ainsi, dans Muliats, Natasha Kanapé Fontaine, une poète et slameuse reconnue dans le monde culturel francophone, interprète des poèmes en langue innue et provoque des effets d'immédiateté inattendus par sa présence. Tandis que la pièce se déroule majoritairement en français, Kanapé Fontaine incarne divers personnages qui s'expriment en langue innue. La récitation des poèmes fait vivre la langue innue hors des réserves, hors des territoires dits « autochtones », tout en déterritorialisant la langue parlée à Muliats, au théâtre et sur scène. En entrevue, Kanapé Fontaine décrit ainsi son travail artistique :

Je n'ai jamais parlé pour moi, dit-elle. Dans mes deux derniers recueils, j’ai écrit au « je », mais je dis « je » pour dire «les autres », afin que nous soyons, que nous devenions, nous, les Autochtones, une collectivité plus forte. Je souhaite que nous retrouvions la plénitude que nous apportait notre relation avec notre environnement, surtout pour les générations qui ont subi une rupture à cause des pensionnats, puis, plus loin encore, à cause de la Conquête et de la colonisation ${ }^{24}$ (Voir la figure 2).

${ }^{22}$ Gilles Deleuze et Félix Guattari, Kafka. Pour une littérature mineure, Paris, Les Éditions de Minuit, coll. « Critique », 1975, p. 3I.

${ }_{23}$ Chiel Kattenbelt, « Theatre as the Art of the Performer and the Stage of Intermediality », dans Freda Chapple et Chiel Kattenbelt (dir.), Intermediality in Theatre and Performance, Amsterdam, New York, Rodopi, 2006, p. 36-37.

24 Nathalie Collard, «Je dis "je" pour dire les autres», La Presse, 5 mars 2016, http://plus.lapresse.ca/screens/o7argfif-ef2a-4f3e-aabd-732737do3c83\%7C_o.html (consultation le ro avril 20i6). 


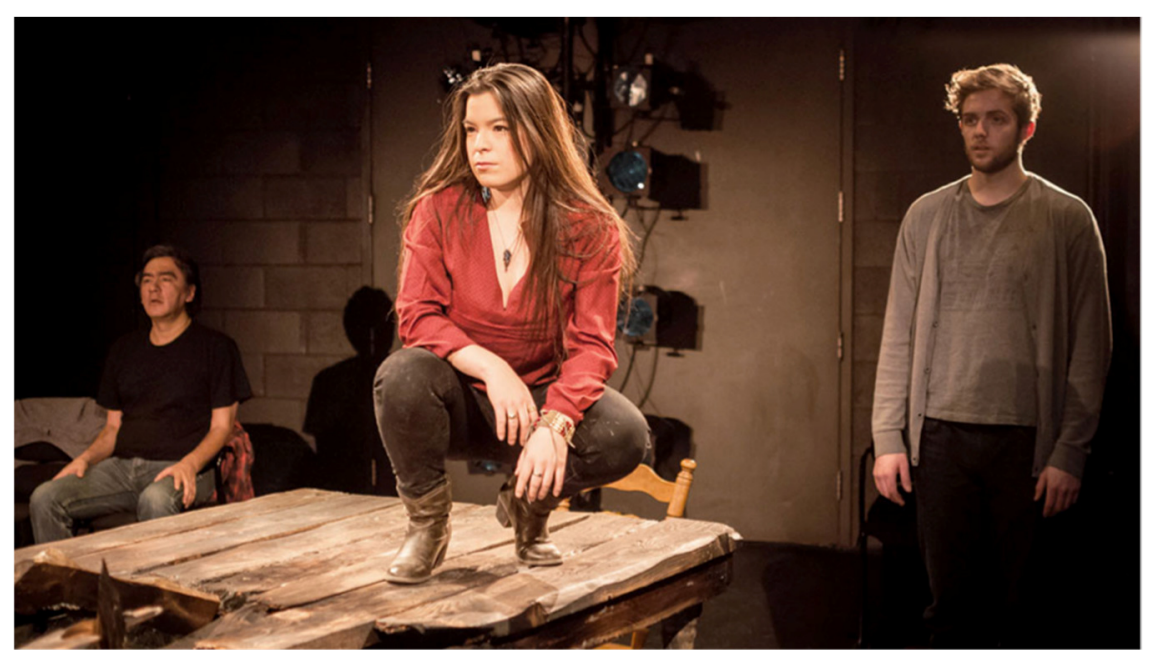

Fig. 2. Résistance poétique en langue innue. Muliats, Productions Menuentakuan et Théâtre Denise-Pelletierb 2016. Photo : Colin Earp-Lavergne.

En assistant à la pièce, j'ai pu remarquer le malaise créé par le basculement du français à l'innu. Marco, le frère de Shaniss, remarque, en riant, que le public ne comprend rien à l'innu et il se met à enseigner quelques mots en innu aux spectateurs :

Marco : Ok. Maintenant, un plus dur ! Serez-vous capables ! PI-SHUM.

Les trois autres comédiens ne comprennent toujours pas ce qui se passe. Tranquillement, ils vont se mettre à participer avec le public.

Public: Pishum.

Marco : Pishum ???

Public: Pishum.

Marco : Yeah ! Vous êtes forts. Pishum, c'est « soleil ». Maintenant, encore plus dur. TE-PESH-KAU. TEPESHKAU.

Même jeu: il fait répéter le public.

Noirceur! Tepeshkau, ça veut dire «noirceur »! Maintenant, répétez après moi ! TE-PESH-KAU PI-SHUM!!! TEPESHKAU PISHUM !!!

\section{Le public répète.}

LUNE !!! Ahah! Tepeshkau pishum, ça veut dire « LUNE »!!! Ahaha! « Le soleil de la nuit ${ }^{25} \gg$ !

Lorsqu'il y a traduction, celle-ci révèle une différence notable entre la signification en langues innue et française de concepts comme le soleil et la lune. C'est un aperçu de ce qui nous attend dans la langue innue. Nous sommes amenés à comprendre, à travers

\footnotetext{
${ }^{25}$ Charles Bender et al., Muliats, texte inédit, 2016, p. 19-20.
} 
l'explicitation par la traduction et l'humour, qu'il est possible de parler du monde en d'autres termes, avec d'autres rythmes, d'autres accents, d'autres nuances et d'autres couleurs. La dynamique langagière de l'intimité hétérolinguale se fonde sur la distance, l'absence, la perte inévitable. Les spectateurs sont interpellés par le contact avec les comédiens, amenés à réagir et à interagir, à s'ouvrir à une nouvelle intimité avec la langue innue, à laisser agir le décentrement de l'espace théâtral et de son langage habituel au profit d'une hétérogénéité linguale et sociale. La traduction met en lumière ce que partagent toutes les langues entre elles. Elles sont à la fois toutes complètes et s'étendent à une réalité qui les dépasse, et, ce faisant, elles sont aussi incomplètes, la traduction n'étant plus comprise comme un acte invisible ou neutre.

Dans Muliats, la rencontre mène à une déterritorialisation pour tous les personnages, mais l'œuvre est en soi déterritorialisante. Il serait facile d'imaginer que la pièce présente d'autres combinaisons de langues et se décline en une multiplicité de rencontres entre des membres de différentes Nations autochtones et des Québécois, des Canadiens, ou même des Américains, des Australiens, des Néo-Zélandais - partout, en somme, où existe un contexte de colonialisme de peuplement.

\section{ACTE D’APPRENTISSAgE ET RÉSISTANCE DES MATÉRIAUX}

En ce sens, on pourrait dire que Muliats propose un apprentissage et constitue un acte de rencontre entre autrui et le « nous » qui habitent le territoire québécois. Le public, divisé en deux, se regarde, à la manière des protagonistes de la pièce. Le décor minimaliste, qui se résume à une imposante table en bois massif, fendue et brûlée en son milieu, avec un vide qui en sépare les deux extrémités, semble reproduire l'écart social entre colons et colonisés. Des jeux d'éclairage accompagnent les multiples instances de chant et de musique traditionnelle de tambour, incarnées par Marco, le frère de Shaniss, qui chante en innu. Le public, dans ce cas montréalais, réparti des deux côtés de la scène, découvre les forces historiques et sociales qui déterminent notre « gouffre » social et voit à quoi pourrait ressembler une communauté, qui partage le territoire de façon décolonisée, où la socitété dominante ne cherche pas à saborder ce qui est senti comme marginal, en ne tentant pas de tout traduire, mais en acceptant les écarts, qu'ils soient linguistiques ou culturels (voir la figure 3 ). 


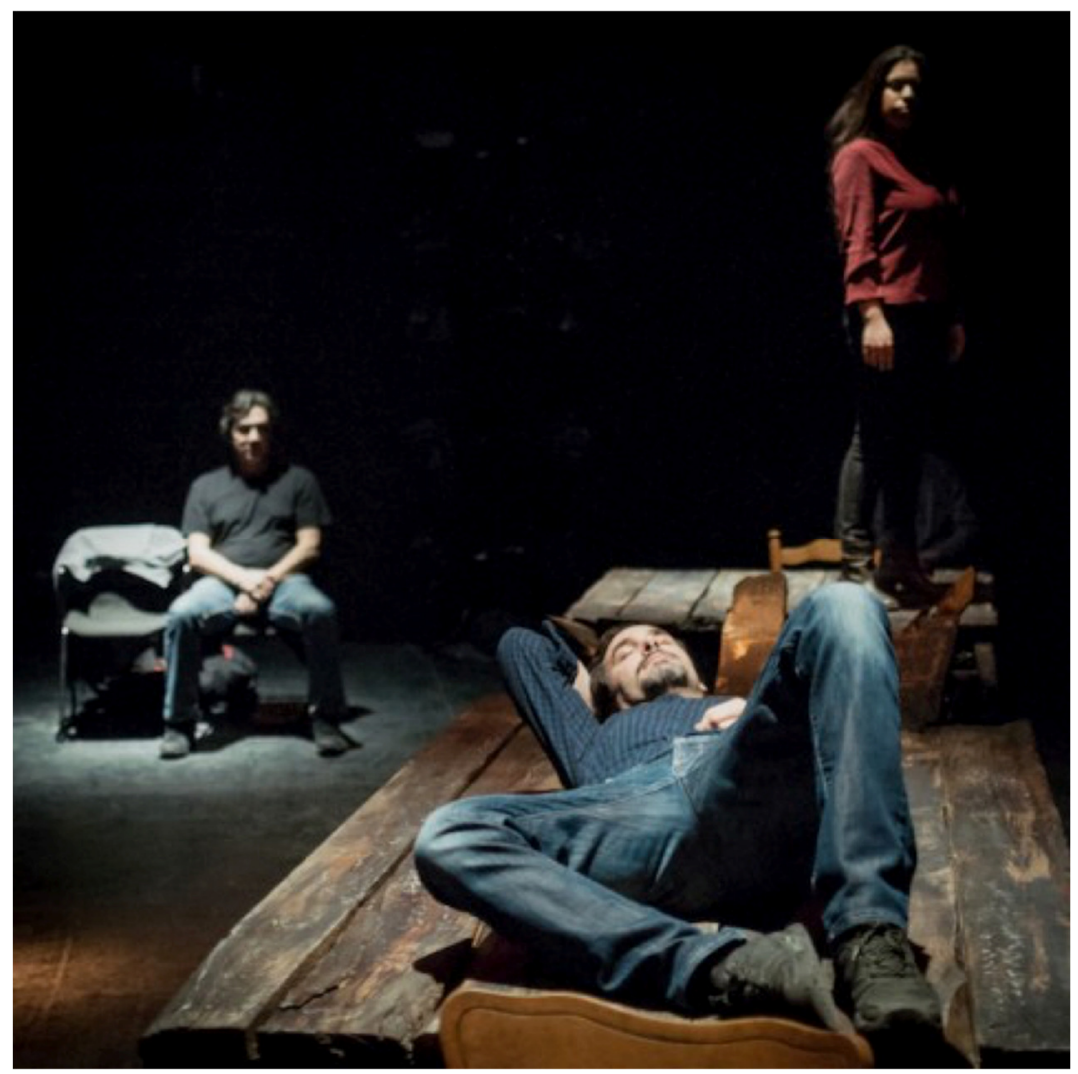

Fig. 3. La table. Muliats, 20ı6. Productions Menuentakuan et Théâtre Denise-Pelletier. Photo : Colin Earp-Lavergne.

Dans une scène, Natasha Kanapé Fontaine se fond dans la peau du personnage d'une jeune femme québécoise qui, en apprenant que son ami Christophe a un colocataire autochtone, se met à déverser un flot de propos racistes, par ailleurs bien connus et répandus, envers les Autochtones :

Raphaëlle: (Soudainement très sérieuse.) Relaxe, calvaire! C'est quoi, là ? T'as décidé que tu prenais leur bord tout d'un coup ? Criss, la moitié de nos taxes va à les faire vivre parce qu'y ont perdu la guerre pis qu'on prend pitié d'eux, pis j'peux pas trouver ça juste un peu cave qu'on fasse ça ? Criss! Quand quelqu'un perd une guerre, y'a pas d'affaire de s'excuser d'avoir gagné pis de leur payer des maisons pis des ski-doos, tabarnak ${ }^{26}$ !

${ }^{26}$ Ibid., p. 23. 
Grâce à ce jeu de Filou (Trickster) ${ }^{27}$, le public est confronté à la violence de sa langue et de son propre discours raciste. Par la voix de Kanapé Fontaine, les spectateurs québécois sont renvoyés à leur reterritorialisation réactionnaire au détriment d'autres aspirations politiques qui peinent à être reçues dans le discours dominant.

La difficile rencontre entre Christophe et Shaniss, entre ceux qui ont subi l'époque du Speak White et ceux qui la subissent encore, se termine par une invitation à se déterritorialiser face à l'hégémonie de la langue française dite « maternelle », à trouver une ligne de fuite commune, à esquiver le pouvoir colonial. Dans Muliats, la colocation entre Shaniss et Christophe, et, ensuite, la rencontre de ce dernier avec Marco amèneront Shaniss et Marco à inviter Christophe à visiter Mashteuiatsh. Shaniss, quant à lui, décide de retourner visiter sa communauté natale, maintenant que le conflit avec son frère à propos de son identité métisse a laissé place à une ouverture. La pièce fait découvrir la possibilité d'échapper à la société dominante en se rendant, ensemble, sur un territoire innu traditionnel ancestral. Ce territoire, bien que soumis à la violence de l'État à bien des égards, demeure un espace de résistance à la colonisation de peuplement.

À la fin de la pièce, le public a droit à une période de discussion avec les comédiens en buvant du thé du Labrador. L'événement donne tout son sens au nom de la compagnie de théâtre, Menuentakuan (« prendre le thé »). Ce faisant, le théâtre traditionnel prend une nouvelle tournure intermédiale, créant un espace de dialogue en temps réel entre les auditeurs et les comédiens autochtones. La rencontre à laquelle sont conviés les spectateurs en buvant du thé est toujours renouvelée : tout n'est pas expliqué une fois pour toutes, chaque représentation de la pièce donnant lieu à une discussion et à un devenir différents. Éric Méchoulan explique à ce propos que « le médium est $[. .$.$] ce qui permet les échanges dans une certaine communauté à la fois$ comme dispositif sensible [...] et comme milieu dans lequel les échanges ont $\operatorname{lieu}^{28} \gg$. Là se situe le caractère politique et décolonisateur de la compagnie Menuentakuan : celle-ci investit l'institution théâtrale qui a historiquement marginalisé les Autochtones. En tant qu'agent d'intermédialité, Muliats soutient l'idée, comme l'atteste Méchoulan, que, si « la relation est par principe première [...], les objets sont avant tout des nouds de relations ${ }^{29} \gg$.

${ }^{27}$ Le Trickster est un héros culturel autochtone qui possède la capacité de changer de forme dans le but de révéler des contradictions et ainsi transformer le monde. Barbara Godard, « Writing Between Cultures », TTR: traduction, terminologie, rédaction, vol. Io, n I, I997, p. 53-99.

${ }^{28}$ Méchoulan, 2003, p. 16.

29 Ibid., p. II. 
Lorsque j'ai assisté à la représentation, le public avait des questions et des hypothèses, notamment à propos de la table, qui, visiblement, résistait à l'interprétation. Certains spectateurs ont parlé de la table fendue comme d'un symbole de l'identité fracturée des Autochtones, du territoire décimé, de l'écart qui sépare les Québécois des Autochtones. Les comédiens ont par contre bien évité de donner une signification à cette table. Ils ont simplement répondu que, lors des représentations de la pièce dans les communautés autochtones - qui ont eu lieu pendant plusieurs années avant d'investir le Théâtre Denise-Pelletier, à Montréal —, les questions étaient complètement différentes. Aussi, les comédiens ont expliqué leur choix de ne pas traduire certaines répliques en langue innue: au départ, la traduction complète en français avait été envisagée, mais ce fut un choix conscient d'éviter la traduction de certaines répliques.

Cet événement-rencontre représente, à mon avis, la mise en œuvre d'un contrediscours politique qui vise à mettre en lumière les tensions sociales entre allochtones et Autochtones par la performance du dialogue, parfois difficile, entre les personnages. L'hybridation linguistique de cette performance nous invite à envisager les différences au sein de l'identité collective comme une résultante de cette rencontre.

\section{AVANT LES RUES: ACTE COLLECTIF DE TRADUCTION}

Alors que Muliats est une ouvre caractérisée par la non-traduction, le film Avant les rues, d'abord présenté dans le cadre du festival Rendez-vous du cinéma québécois (RVCQ) en 20I5, est une œuvre de double traduction en plus d'être la première fiction réalisée en langue atikamekw au Québec. Shawnouk, un jeune homme de Manawan, tue accidentellement un homme lors d'un vol à main armée. Après s'être enfui et avoir erré dans le bois pendant plusieurs jours, hanté par son acte, il revient dans sa communauté. Là, il fait une tentative de suicide avant de décider de retourner dans le bois pour entreprendre un parcours thérapeutique traditionnel. Aidé par une aînée et un groupe d'hommes, il participe à des cérémonies spirituelles traditionnelles. Ce retour aux traditions ancestrales lui procurera, en quelque sorte, la voie de sa libération (voir la figure 4$)^{30}$.

${ }^{\circ} \mathrm{La}$ bande-annonce est disponible sur le site web du film www.Avantlesrues.com (consultation le 25 mai 20I6). 


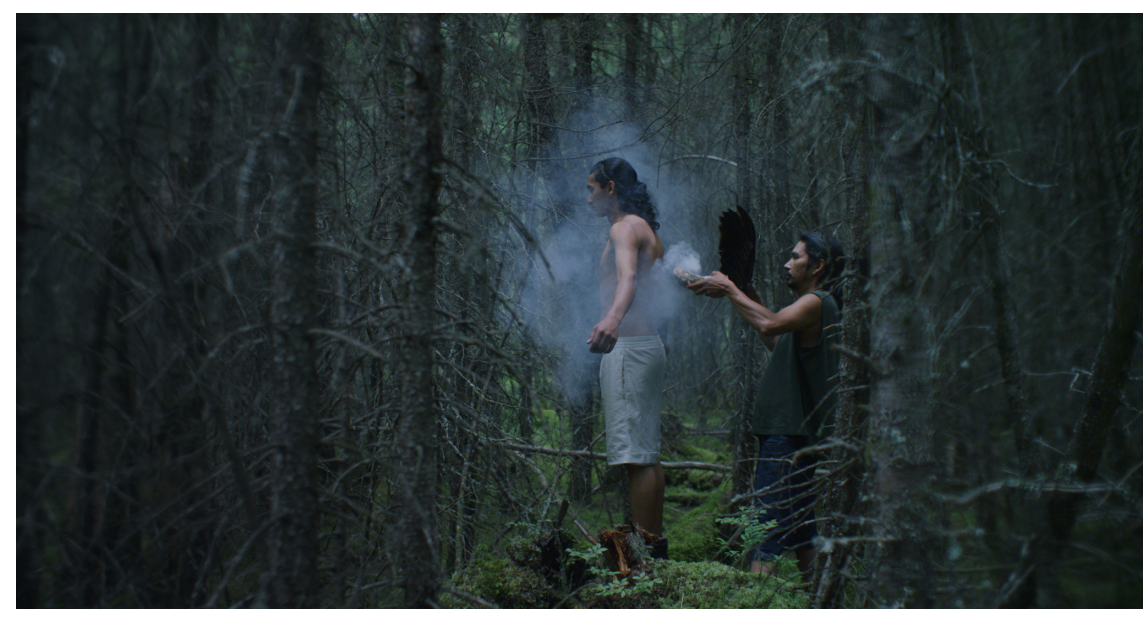

Fig. 4. Préparation à un pow-wow. Photogramme du film Avant les rues, Chloé Leriche, 2016.

Le scénario a d'abord été créé en français par Chloé Leriche, une cinéaste autodidacte non autochtone qui a collaboré au projet du Wapikoni mobile ${ }^{3 \mathrm{I}}$. Si plusieurs cinéastes québécois ont déjà réalisé des films à propos des Autochtones ${ }^{32}$, Avant les rues se démarque par le travail de réalisation collaboratif avec les comédiens atikamekw. Les dialogues en français ont été traduits collectivement en atikamekw par les comédiens eux-mêmes. À la fin du tournage, les dialogues ont été retraduits en français pour assurer le sous-titrage du film. Le travail collectif de traduction opère ainsi un retour au français, mais les sous-titres ne renvoient pas nécessairement au texte original de Leriche. En entrevue ${ }^{33}$, Chloé Leriche explique avoir voulu mettre de l'avant le rôle de la langue comme base de la culture.

La particularité du processus réside dans le fait que Leriche ne parle pas l'atikamekw; elle a donc dû confier aux comédiens le travail de traduction des répliques. Les comédiens ont par ailleurs pu improviser les scènes à divers degrés, et c'est là qu'a eu lieu le travail de réappropriation du médium cinématographique. Lorsque, après le

${ }^{31}$ Le Wapikoni mobile met, depuis les années 2000, des studios de production de films ambulants à la portée de jeunes autochtones de trente communautés au Québec afin de produire des courts métrages, www.wapikoni.ca/ (consultation le 25 mai 20ı6).

${ }^{32}$ Cornellier, 20II, p. 92-II3.

33 Karina Chagnon, «Entrevue avec Chloé Leriche», Trabir, I2 avril 2016, https://trahir.wordpress.com/2016/04/12/chagnon-leriche/ (consultation le 3 mai 2016). 
tournage, la réalisatrice a commencé à faire le montage des scènes à partir du texte à l'aide de la linguiste atikamekw Marie-Pier Ottawa, elle raconte s'être aperçue des nuances qu'avaient apportées à son texte les comédiens à travers l'improvisation en langue atikamekw, et elle a ensuite cherché à les mettre en lumière ${ }^{34}$. Leriche mentionne notamment la notion de respect qui a été ajoutée dans une scène. À la suite de la tentative de suicide de Shawnouk, sa copine vient lui parler. Elle lui demande: «Qu'est-ce que t'as fait de ton respect35 ? » Cette notion de respect ne figurait pas dans le texte original. Leriche dit n'avoir jamais considéré l'association entre les notions de respect et de mort. Mais elle explique que la notion de respect de soi, associée à un concept de fierté, est fondamentale dans la culture autochtone, participant à une ontologie totalisante. Comme pour d'autres écarts entre le texte original en français et les scènes traduites et improvisées en atikamekw, Leriche a conservé cette improvisation de la part des comédiens.

\section{INTIMITÉ HÉTÉROLINGUALE}

En tant qu'action humaine, la traduction a nécessairement une dimension politique. Pendant l'ère moderne coloniale, la traduction a participé à la construction d'une langue liée à l'identité nationale, et la traduction s'est associée à un processus d'homogénéisation identitaire et linguistique. Par la traduction, on a construit des schémas de représentation du sujet. C’est ce que Jon Solomon nomme la biopolitique de la traduction, qui « [...] désigne cet espace d'échange et d'accumulation dans lequel le politique est apparemment préempté par l'occurrence de la langue ${ }^{36} \gg$. Solomon explique que le phénomène moderne de standardisation linguistique est intimement lié à la nationalisation et à l'appropriation de terres colonisées: le projet de standardisation, d'abord linguistique, finit par s'étendre à toutes les formes de différences sociales dans un processus d'homogénéisation nationale et de segmentation interne. Ce rapport entre le nationalisme impérial et les minorités ethniques fait en sorte que la traduction peut être comprise comme un schéma de représentation du sujet épistémologique dans lequel ce dernier est classifié sur une base nationale ou encore

\footnotetext{
${ }^{34}$ Ibid.

35 Avant les rues, Chloé Leriche, 2016.

${ }^{36}$ Solomon, 2007, para. 4.
} 
raciale ${ }^{37}$. Ainsi, seuls les autres ont des « cultures ». De son côté, la philosophe Rada Iveković $3^{8}$, dans son texte sur les enjeux sociaux et culturels de la traduction, décrit comment « l'histoire coloniale a fait éclater au grand jour les tensions politiques du traduire, de même qu'elle a fait apparaître et exposé les différents points de vue, les contextes et perspectives décentrés, contradictoires, pluriels et hors champ ${ }^{39} \gg$. À l’ère postcoloniale ou encore postmoderne :

La traduction tisse l'imprescriptible du droit d'asile universel et le droit universel à la déterritorialisation, reterritorialisation, mais principalement à la déprise de l'ancrage fatal dans quelque identité que ce soit. Elle permet de construire le non-destin du vivant, elle permet de se déprendre de la théologie de l'Occident, ou de l'Un, ce qui historiquement revient au même tout en ne relevant d'aucune métaphysique ${ }^{40}$.

Dans la notion de l'intimité hétérolinguale par la traduction, Jon Solomon met de l'avant l'idée que l'intimité sociale fait obstacle à la traduction par l'adoption d'une langue non maternelle et par l'exigence que les locuteurs de langue maternelle reconnaissent les accents étrangers à l'intérieur de celle-ci ${ }^{41}$. Les contraintes d'espace et de temps associées à la lecture des sous-titres, propres au médium cinématographique, ont forcé Leriche à opter pour un français plus normatif. Elle a donc choisi d'intégrer des mots familiers ou encore des expressions étrangères qui déterritorialisent le français. Par exemple, lorsque Shawnouk parle avec l'aînée, son discours prend des tournures inédites. Il dit à l'aînée : « Merci de m'avoir fait manger » et « Merci de m'avoir fait dormir $^{42} \gg$. Cette traduction non idiomatique est une tentative de relever la distance qu'exige la déférence d'un jeune lorsque celui-ci s'adresse à une aînée. À l'opposé de la normalisation linguistique et des divisions géoculturelles homogénéisantes, Solomon propose une forme de déterritorialisation qui mélange le commun et « l'étranger » dans la langue pour se mobiliser contre la violence biopolitique contemporaine ${ }^{43}$. Le processus de double traduction collective du scénario de Leriche produit ainsi des effets de langue qui se démarquent considérablement des traductions que Brisset appelle

37 Ibid.

${ }^{38}$ Rada Iveković, «Que veut dire traduire? Les enjeux sociaux et culturels de la traduction », Revue Asylon(s), n 7, 2009, http://www.reseau-terra.eu/article889.html (consultation le 3 mai 2016).

39 Ibid., para. 3.

40 Ibid., para. 24.

${ }^{41}$ Solomon, 2007, para. I3.

${ }^{42}$ Avant les rues, Chloé Leriche, 2016.

${ }^{43}$ Solomon, 2007, para. II. 
« identitaires ${ }^{44}$ » et qui ont caractérisé à une époque la traduction d'œuvres théâtrales au Québec. L'intérêt de la traduction ne se situe plus dans une transmission de la parole «étrangère » pour nourrir son propre discours (québécois), mais réside dans une traduction qui révèle la réalité colonisée, marginalisée, minorisée en démontrant «l'impossible d'une grande culture nationale45 ».

Comme c'est le cas dans la pièce Muliats, les paroles de Shawnouk ne sont pas celles d'un seul personnage, elles relèvent plutôt d'un agencement collectif d'énonciation. Son personnage est branché sur l'immédiat-politique; sa tentative de suicide est d'autant plus troublante que la sortie du film en salle a coïncidé avec une vague de suicides de jeunes Autochtones dans plusieurs réserves à travers le Canada qui a fait les manchettes. Contrairement au discours dominant dans les médias, qui offre encore comme seule solution l'assimilation - pensons ici aux propos tenus par l'ancien ministre des Affaires indiennes et ancien premier ministre Jean Chrétien ${ }^{46}$-, Avant les rues mise sur le retour au mode de vie traditionnel. Déjà, le titre du film en atikamekw, Tewebikan epwamoci meskanawa, évoque ce parti pris : il signifie « Le tambour qui était avant les rues ». Le terme «tewekikan » signifie «tambour », et il contient le mot «bikan», « cour ». En atikamekw, le tambour évoque le premier battement du cœur, le premier battement de la vie. Plus qu'une invocation à la nostalgie de la vie avant la colonisation, Tewehikan epwamoci meskanawa appelle à un avenir individuel et collectif qui se fonde dans la tradition comme source de libération et de guérison (voir la figure 5).

${ }_{44}$ Annie Brisset, 1990, p. I49.

45 Dalie Giroux, «Homi Bhabha et le Québec : appel à un "acte insurgeant de traduction culturelle” », Spirale, Montréal, no 258, automne 2016, p. 40.

${ }^{46}$ Jean Chrétien, lorsqu'il a été interrogé sur la vague de suicides à Attawapiskat a répondu «Prenez le nord du Manitoba, c'est extrêmement difficile d'avoir une vie là-bas. Mais les autochtones sont traditionalistes. Ils veulent rester près de leur terre, ils ont la nostalgie du passé, quand ils vivaient de chasse et de pêche [...] C'est bien de rester s'ils le veulent, mais ce n'est pas toujours possible [...] Il y a des fois où ils devraient déménager, comme n'importe qui d'autre. » Lysiane Gagnon, «Réserves: rester ou déménager?», La Presse, I9 avril 2016, http://www.lapresse.ca/debats/chroniques/lysiane-gagnon/201604/I8/oI-4972492-reservesrester-ou-demenager-php (consultation le $\mathrm{I}^{\mathrm{er}}$ novembre 20I6). Cet appel à simplement « déménager » est constamment répétée lorsque vient le temps de faire face aux conditions de vie des Autochtones dans les réserves. Plutôt que reconnaître le sous-financement des services, l'héritage des pensionnats et autres politiques gouvernementales comme la source de ces problèmes sociaux, les gouvernements maintiennent que quitter le territoire ancestral est la seule solution. 


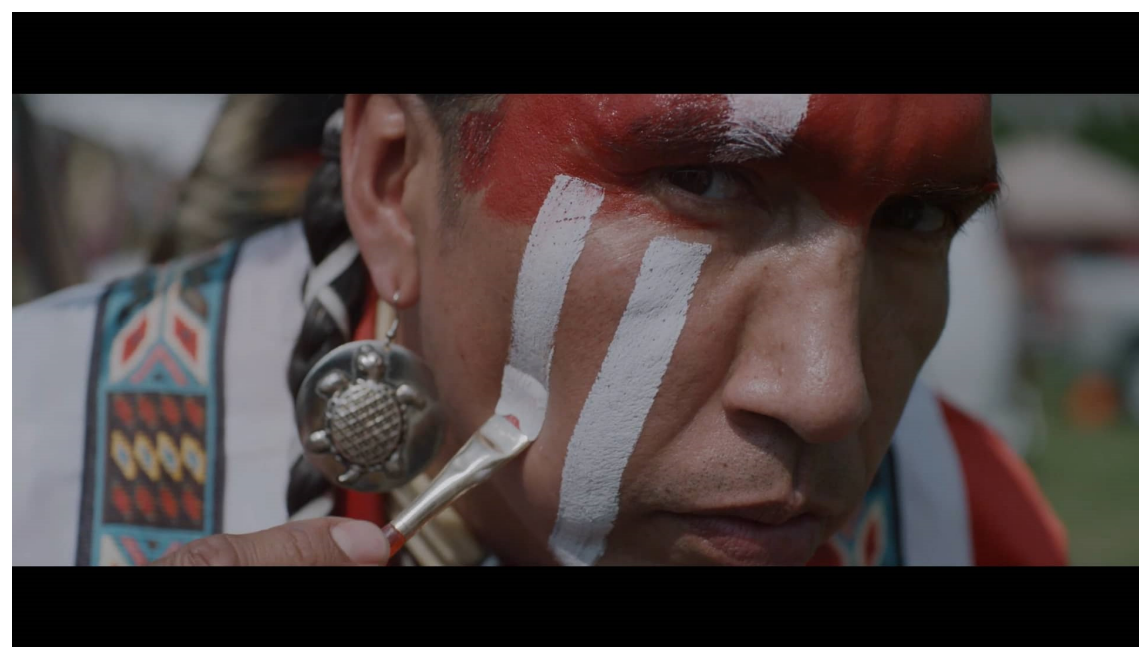

Fig. 5. Préparation à un pow-wow. Photogramme du film Avant les rues, Chloé Leriche, 2016.

D'ailleurs, le chant et le tambour sont d'une importance centrale dans le film. En effet, les comédiens qui interprètent Shawnook et sa sœur sont des chanteurs de musique traditionnelle autochtone et font partie du groupe Northern Voice ${ }^{47}$. Or, dans tout le film, seules les paroles des chansons n'ont pas été traduites. Leriche explique ce choix par une volonté de rendre au spectateur une émotion qui ne soit pas embarrassée par la signification. Le cinéma permet en effet cette possibilité en tant que média qui peut accueillir, par l'image, un son et une parole qui en émane (voir la figure 6).

Dans Avant les rues, les images et les sons jouent avec des seuils d'intensité pour nous faire découvrir une forme de vie liée au territoire. Les images du film expressionniste font usage d'un double mouvement de déterritorialisation et de reterritorialisation. Le territoire, au cœur de cet univers, se présente comme des lieux que les non-Autochtones connaissent bien - des chemins en terre battue aux abords de la communauté dans la région de Lanaudière, des carrières de sable, des chemins forestiers ornés de minces lisières d'arbres qui cachent des coupes à grande échelle, des paysages enlevants de lacs bordés de montagnes. Mais, dans le retour aux formes de vie atikamekw, le territoire revêt une dimension sacrée étrangère au public québécois : c'est au cour de la forêt qu'ont lieu la cérémonie de purification, le cercle de guérison, le

\footnotetext{
47 Tribal Spirit Music, 2013, «Northern Voice Singers », http://www.northernvoicesingers.com/ (consultation le 2 mai 20r6).
} 
passage dans une hutte de sudation, le tambour et le chant, tous des éléments qui amènent Shawnouk à guérir de ses blessures.

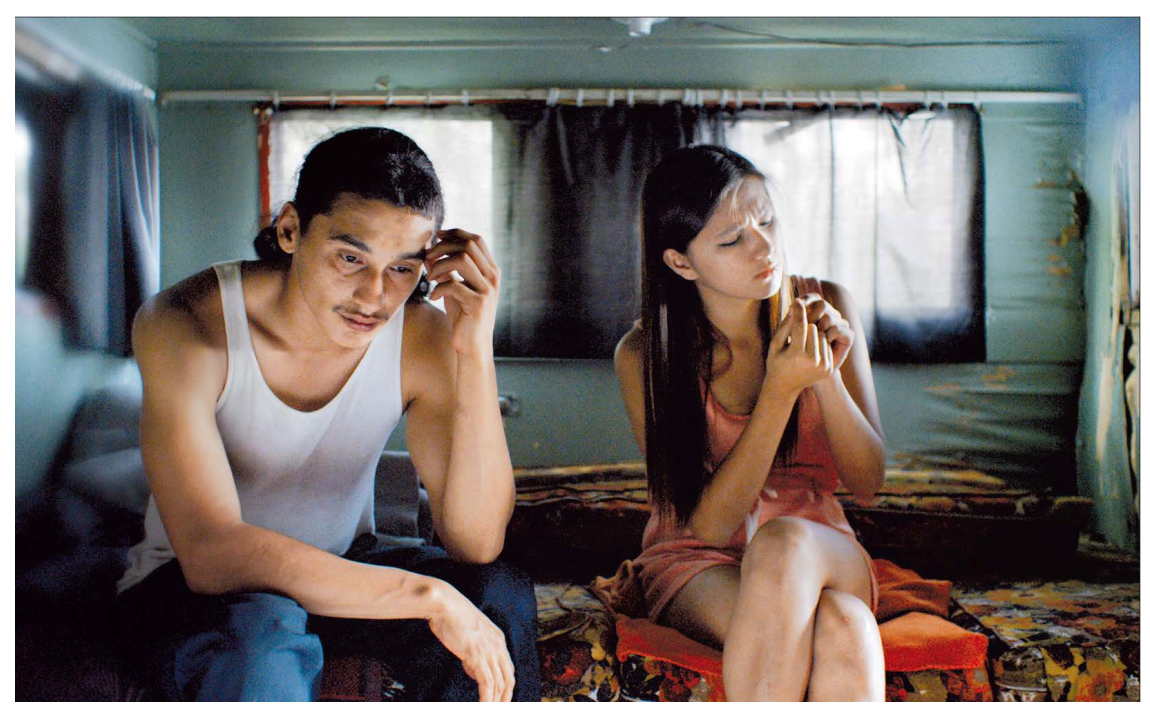

Fig. 6. Les comédiens Rykko Bellemare et Kwena Bellemare Boivin, chanteurs du groupe Northern Voice. Photogramme du film Avant les rues, Chloé Leriche, 2016.

Il n'en demeure pas moins qu'en raison de l'opacité propre au médium cinématographique, qui tend à présenter l'illusion d'une réalité transparente en masquant ses propres aspects, le film demeure une instance moins réflexive que le théâtre ${ }^{8}$. Outre les quelques possibilités de rencontrer les comédiens en présence de la réalisatrice lors de la tournée promotionnelle du film, les auditeurs sont moins bouleversés par la complexité de la performance. Malgré la collectivisation du travail cinématographique, le processus n'est pas explicité dans sa mise en marché, et c'est à la réalisatrice que reviennent les honneurs d'un travail auquel ont participé de nombreux agents linguistiques, dont les comédiens, mais aussi les traducteurs et les sous-titreurs. De ce point de vue et au regard de nos deux exemples, les modalités propres au théâtre semblent mieux disposées à mettre de l'avant l'aspect collectif des œuvres ainsi que la possibilité de créer des espaces de dialogue.

\footnotetext{
${ }^{48}$ Kattenbelt, 2006, p. 34.
} 


\section{LA DÉCOLONISATION PAR LA TRADUCTION?}

La méthodologie de décolonisation, qui met l'accent sur des valeurs de réciprocité entre chercheurs et collectivités autochtones, souligne le rôle central du travail collectif pour éviter les représentations fétichisantes de la part des chercheurs à propos de leurs « sujets ethnologiques ». À ce titre, nous pourrions comprendre le travail collectif des Productions Menuentakuan et de Chloé Leriche avec leurs comédiens respectifs comme des tentatives d'échapper aux rapports dichotomiques entre soi et l'autre. Ce sont plutôt les rapports entre les comédiens, réalisateurs et metteurs en scène qui créent des effets de décentrement de la langue dominante. Comme Muliats, le film Avant les rues met en acte, dans le discours, un agencement collectif d'énonciation, où la traduction et la non-traduction des dialogues participent de postures politiques. Dans les deux ouvres étudiées, j'ai tenté de comprendre comment le tissage des langues innue et atikamekw avec le français a participé à des démarches d'appropriation des modalités théâtrale et cinématographique, et à la création d'espace de contre-discours.

Il reste cependant à étudier le positionnement de ces projets dans la sphère artistique. Le cas de la littérature et, plus généralement, de l'art autochtone au Québec révèle une double exiguïté, ce qui explique peut-être pourquoi de telles expressions artistiques sont si peu présentes dans l'univers francophone québécois comparativement à l'univers anglo-saxon canadien. D'ailleurs, les études autochtones (Indigenous Studies), très populaires dans le monde anglophone canadien, commencent à peine à faire leur entrée dans la sphère francophone. Osons croire qu’à l'aide de telles œuvres politiques hétérolinguales, nous serons amenés à repenser nos rapports politiques entre Autochtones et allochtones au Québec. 


\title{
Muliats et Avant les rues: la politique de l'œuvre hétérolinguale
}

\author{
Karina Chagnon, Université du QuÉBEC À MONTRÉal
}

\section{RÉSUMÉ}

Ce texte propose d'étudier deux œuvres récentes, la pièce de théâtre Muliats des Productions Menuentakuan (2016) et le film Avant les rues de Chloé Leriche (2016). Ces œuvres collectives de traduction et de non-traduction permettent une réflexion sur l'appropriation de différents dispositifs intermédiaux en vue de faire exister, dans l'espace public, les langues innue et atikamekw dans une intimité hétérolinguale avec le français québécois.

\section{ABSTRACT}

This article analyzes two recent cultural productions, the play Muliats by Productions Menuentakuan (2016) and the film Avant les rues by Chloé Leriche (2016). These productions, both of a collective nature, offer a mixture of translation and nontranslation. Different intermedial tools are appropriated as a way of bringing the Innu and the Atikamekw Indigenous languages into the public sphere as they are woven with Québécois French to create an intimate form of heterolingualism.

\section{NOTE BIOGRAPHIQUE}

Karina Chagnon est traductrice professionnelle et doctorante en sémiologie à l'Université du Québec à Montréal. Son projet de recherche porte sur les rapports entre langue et traduction dans la dé/colonisation au Québec et au Canada. Elle dirige actuellement le chantier de recherche « Traduire les humanités » avec le Laboratoire de résistance sémiotique de l'UQAM et contribue aux revues Trabir, Spirale et Cygne noir. 